\title{
Retraction Notice to: New Solution Generation Strategy to Improve Brain Storm Optimization Algorithm for Classification
}

\author{
Yu Xue ${ }^{1,2, *}$ and Yan Zhao ${ }^{1}$ \\ ${ }^{1}$ School of Computer and Software, Nanjing University of Information Science and Technology, Nanjing, China \\ ${ }^{2}$ Jiangsu Key Laboratory of Data Science and Smart Software, Jinling Institute of Technology, Nanjing, China
}

The published article titled "New Solution Generation Strategy to Improve Brain Storm Optimization Algorithm for Classification" has been retracted from the Journal on Internet of Things, Vol.3, No.3, 109$118,2021$.

DOI: 10.32604/jiot.2021.014980

URL: https://www.techscience.com/jiot/v3n3/46020/pdf

After the publication of the above article, the authors Xue et al. declare that due to the following reasons, they have to retract the published article from Journal on Internet of Things:

1. The authors need to conduct more indepth experiments to verify the proposed method regarding problematic experimental setup, which leads to an unfair comparison with the comparison algorithm.

2. The authors concluded that some error data did not correspond to its true dataset. Algorithm data needed to be adjusted to prevent difficulties for researchers reading the results of the experiments.

After a thorough review of the manuscript content alongside the new disclosures brought to light and in discussion with the editors and the leadership of TSP's Ethics team, the editors and TSP agreed that the significant errors that have been disclosed could not be corrected. Hence, according to the COPE Retraction Guidelines, there is "clear evidence that the findings are unreliable, either as a result of major error." Therefore, this paper should be retracted to maintain academic integrity.

We would like to apologize for the oversight and apologize to our readers for any misleads or inconvenience this may have caused. 